\title{
Inhibition Effect of Organic Electron-Accepting Compounds on Corrosion of Iron in Acid Solution
}

\author{
Kunitsugu Aramaki* \\ *Faculty of Science and Technology, Keio University
}

\begin{abstract}
In order to confirm the adsorption of organic corrosion inhibitors on metals by means of back-donation of the metal electrons to an vacant orbital of the inhibitor, the inhibition efficiency of electron-accepting inhibitors was determined for pure iron in a $6.1 \mathrm{M} \mathrm{HCl}$ solution by polarization measurements. Nitrobenzene derivatives were used as the electron-cacepting inhibitors. They accelerated the cathodic reaction because of their reduction, whereas they inhibited the anodic reaction. The inhibition efficiency of these inhibitors for the anodic reaction was expressed by the Hammett-like equation associated with the Hammett constant, $\sigma$. The efficiency of the compounds with an electron-attracting substituent $(\sigma>0)$ increased with a decrease in the electron density of the nitro group. Since the efficiency of these compounds was closely related to the half-wave potential of reduction, the back-donation of metal electrons to the antibonding $\pi$ orbitals was concluded to play an important role in the adsorption of the nitrobenzene derivatives on the iron surface.
\end{abstract}

\section{Introduction}

It is generally accepted that organic corrosion inhibitors adsorb on metallic surface by means of three principal types of interaction between the inhibitor molecule and the metal ${ }^{11}$. They are an electrostatic, a covalent, and a $\pi$-bonding interactions. An ionic inhibitor like a quaternary ammonium cation adsorbs on the negatively charged surface by the electrostatic force. Most of polar organics chemisorb on the metal by the formation of covalent bond between the polar atom and metal. In general, a pair of unshared electrons in the polar atom is donated to a vacant d-orbital of the metal. A flat adsorption of aniline and allyl amine, oriented in parallel to the surface, occurs by $\pi$-electron bonding between the metal and the phenyl and the allyl groups $^{2), 3)}$. Therefore, the inhibition efficiency of aniline derivatives and olefinic amines is related not only to the electron density of nitrogen atom but also to the $\pi$-electron density of the phenyl group and the double bond. The $\pi$-bonding interaction also relates to back-coordination of metal electrons to a vacant orbital of polar atom. For example, tetra-n-propylsilane $\left(\mathrm{C}_{3} \mathrm{H}_{7}\right)_{4} \mathrm{Si}$, which

* 3-14-1, Hiyoshi, Kohoku-ku, Yokohama, 223 Japan holds no pair of unshared electrons, inhibited the cathodic reaction of various transition and nontransition metals in an acid solution ${ }^{4), 5}$. This result has been explained by occurrence of the back-donation of metal electrons to the vacant d-orbital of $\mathrm{Si}$ atom. The back-donation of metal electrons to an antibonding $\pi$-orbital of the olefinic compounds has also been suggested, since a decrease in the density of $\pi$-electron enhanced the $\pi$-electron adsorption of double bond on the cathode ${ }^{(), 7)}$.

The present work was undertaken to elucidate a possibility of the adsorption by the formation of back-donation linkage between the metal and electron-accepting inhibitor. The inhibitors used were nitrobenzene derivatives which are the electron acceptors for charge transfer complexes. Inhibition efficiency of the inhibitors was determined on pure iron in a $6.1 \mathrm{M} \mathrm{HCl}$ solution by polarization measurements, and discussed with respect to the substituent constant, the electron affinity, and the molecular size of these compounds.

\section{Experimental}

\subsection{Inhibitors}

Eleven ring-substituted nitrobenzene derivatives, listed in Table 1, were used as the inhibitors. They were obtained as high-grade commercial 
Table 1 Effectively covered area, $S$ and polarization data at the inhibitor concentration of $1 \times 10^{-8} \mathrm{M}$.

\begin{tabular}{|c|c|c|c|c|}
\hline No. & Inhibitor & $S\left(\mathrm{~A}^{2}\right)$ & $E_{\text {corr }}(\mathrm{mV})$ & $b_{a}(\mathrm{mV} / \log i)$ \\
\hline 0 & none & - & -467 & 109 \\
\hline 1 & nitrobenzene & 51.6 & -461 & 112 \\
\hline 2 & o-dinitrobenzene & 61.3 & -455 & 110 \\
\hline 3 & $\mathrm{~m}$-dinitrobenzene & 64.3 & -433 & 113 \\
\hline 4 & p-dinitrobenzene & 64.2 & -440 & 112 \\
\hline 5 & $1,3,5$-trinitrobenzene & 77.7 & -422 & 110 \\
\hline 6 & p-chloronitrobenzene & 59.4 & -448 & 111 \\
\hline 7 & 2,4-dinitrofluorobenzene & 66.4 & -437 & 112 \\
\hline 8 & $\mathrm{p}$-nitrotoluene & 58.3 & -450 & 112 \\
\hline 9 & p-nitroanisole & 61.6 & -449 & 112 \\
\hline 10 & p-nitrophenol & 55.2 & -449 & 110 \\
\hline 11 & p-nitroaniline & 60.0 & -446 & 110 \\
\hline
\end{tabular}

reagents and purified by distillation or recrystallization before use. The inhibitors were dissolved in the $6.1 \mathrm{M} \mathrm{HCl}$ solution prepared by diluting reagent-grade concentrated $\mathrm{HCl}$ with redistilled water.

\subsection{Polarization measurements}

Corrosion rates of a $99.89 \%$ iron electrode* (Mallinckrodt analytical reagent grade) were determined in the inhibited and uninhibited $\mathrm{HCl}$ solutions by galvanostatic polarization measurements. The inhibitor solution was deaerated by passing nitrogen gas and kept at $30 \pm 0.1^{\circ} \mathrm{C}$. The cathodic and anodic polarization runs were carried out independently. The details of the measurements have been given elsewhere ${ }^{8}$.

\subsection{Determination of effectively covered area}

The area of metal surface effectively covered by an inhibitor molecule, $S$ was determined by arranging the Stuart-Briegleb molecular models on a plane in the position corresponding to the appropriate conformation and orientation mode of the molecule adsorbed on the surface. The nitrobenzene derivatives were assumed to adsorb in parallel with the surface and not to rotate on the surface. The determination of the area has been described in more detail elsewhere ${ }^{\theta}$.

\section{Results}

Typical polarization curves of the iron electrode in the uninhibited solution and the inhibited solution with nitrobenzene are shown in Fig. 1, indicating acceleration of the cathodic reaction in the presence of nitrobenzene. All the curves showed that the nitrobenzene derivatives stimu-

* Impurities: C, $0.03 \%$; Mn, $0.04 \% ; \mathrm{P}, 0.002 \%$; Si, $0.001 \%$; S, $0.02 \%$.

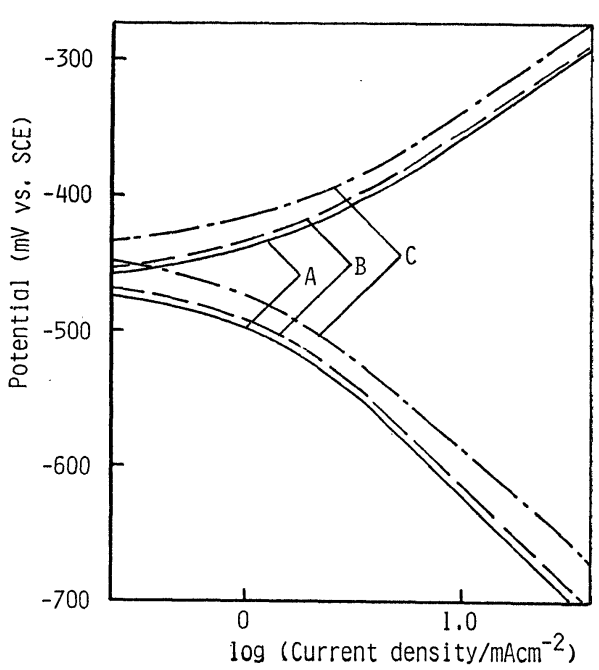

Fig. 1 Polarization curves of iron electrode in $6.1 \mathrm{M} \mathrm{HCl}$ uninhibited (A) and inhibited with nitrobenzene at $1 \times 10^{-3} \mathrm{M}(\mathrm{B})$ and at $3 \times 10^{-3} \mathrm{M}(\mathrm{C})$.

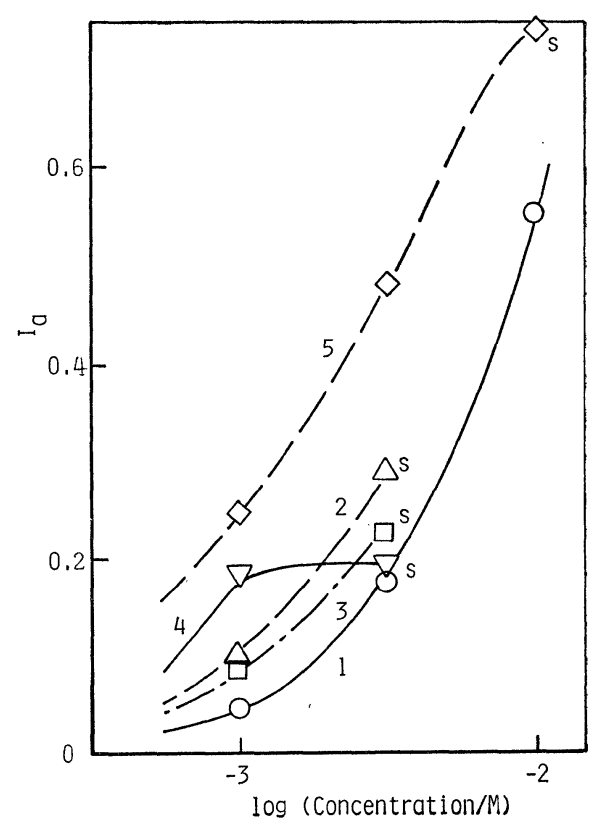

Fig. 2 Anodic inhibition efficiency, $I_{a}$ of nitrobenzene derivatives (1).

1 , nitrobenzene; 2 , o-dinitrobenzene; 3 , m-dinitrobenzene; 4 , p-dinitrobenzene; 5 , 1,3,5-trinitrobenzene. $\mathrm{s}$ : saturated in the solution.

lated the cathodic reaction. This stimulation is attributed to the electrochemical reduction of the nitro compounds to hydroxylamines and finally to amines. Nitrobenzene is cathodically reduced to aniline in an acid medium ${ }^{10)}$, as 


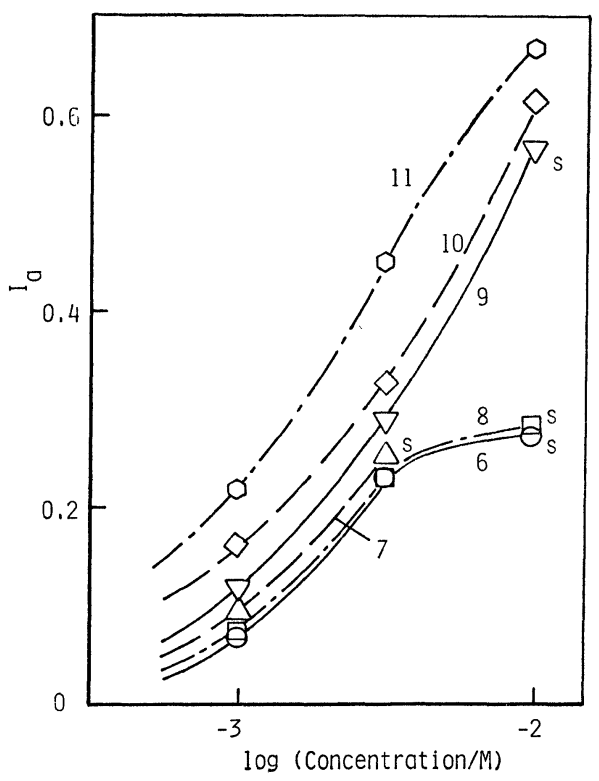

Fig. 3 Anodic inhibition efficiency, $I_{a}$ of nitrobenzene derivatives (2).

6, p-chloronitrobenzene; 7, 2,4-dinitrofluorobenzene; 8 , p-nitrotoluene; 9, pnitroanisole; $10, \mathrm{p}$-nitrophenol; $11, \mathrm{p}$ nitroaniline. $s$ : saturated in the solution.

\section{$\mathrm{C}_{6} \mathrm{H}_{5} \mathrm{NO}_{2} \stackrel{+4 \mathrm{e}+4 \mathrm{H}^{+}}{\longrightarrow} \mathrm{C}_{6} \mathrm{H}_{5} \mathrm{NHOH} \stackrel{+2 \mathrm{e}+2 \mathrm{H}+}{\longrightarrow} \mathrm{C}_{6} \mathrm{H}_{5} \mathrm{NH}_{2}$}

Therefore, the inhibition effect of the adsorbed molecule on the cathodic reaction could not be determined in this experiment.

The nitro compounds, however, inhibited the anodic reaction of the iron electrode in the acid solution. The well-defined anodic Tafel lines were observed in the potential region between -280 and $-380 \mathrm{mV}$ (vs. SCE). The corrosion potential for the inhibited electrode shifted toward the less noble potential by less than $90 \mathrm{mV}$ from that for the uninhibited electrode, $E_{\text {corr }}^{0}$ The potential, $E_{\text {corr }}$ and the anodic Tafel slope, $b_{a}$ at the inhibitor concentration of $1 \times 10^{-3} \mathrm{M}$ are listed in Table 1. There was no significant spread of anodic Tafel slopes. The effectively covered area, $S$ of the inhibitors is also tabulated in the table.

On the assumption that the values of $b_{a}$ are constant, the anodic inhibition efficiency, $I_{a}$ is given by

$$
I_{a}=1-i_{a} / i_{\mathrm{corr}}^{0}
$$

where $i_{a}$ is the current density obtained by extrapolating the anodic Tafel line at $E_{\text {corr }}^{0}$ and $i_{\text {corr }}^{0}$ is the corrosion current density obtained by intersection of the extrapolated anodic and cathodic Tafel lines for the uninhibited electrode.

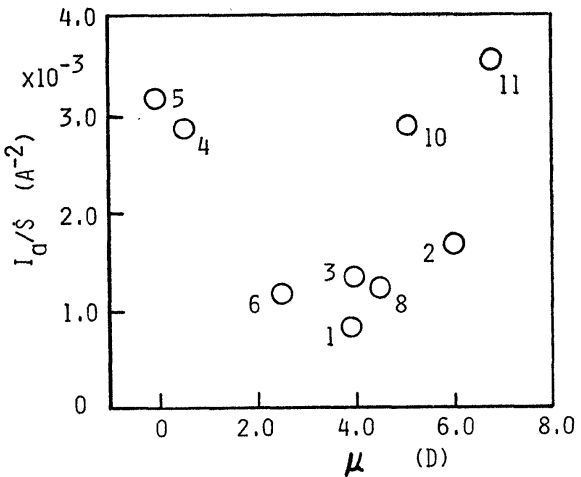

Fig. 4 Relationship between $I_{\alpha} / S$ and dipole moment of inhibitors, $\mu$. The numbers refer to the inhibitors in Table 1.

Fig. 2 and 3 show the anodic inhibition efficiency of the nitrobenzene derivatives.

\section{Discussion}

The nitrobenzene derivatives are effective inhibitors for the corrosion of titanium in acid media ${ }^{11)}$. These compounds are electrochemically reduced on the titanium surface, which leads to passivation of the metal. The corrosion potential of $\mathrm{Ti}$ shifts towrad the noble potential by several hundreds milivolts by the addition of the effective inhibitors ${ }^{12)}$. Rosenfeld has been described that amine nitrobenzoate and 3,5dinitrobenzoate shifted the potential beyond the passivation potential, and thus facilitated passivation of iron in neutral media ${ }^{13}$.

In order to confirm no reduction of the nitro compounds during the anodic polarization measurement in this experiment, UV spectra of the inhibitor solution were determined at the concentration of $1 \times 10^{-3} \mathrm{M}$. The concentration of the nitro compound was decreased during the cathodic polarization, while no change in the concentration was observed during the anodic one. The effect of the reduction on the anodic Tafel line was estimated to be negligible. The reduction potential of the nitro compounds examined in this study was less noble than the potential range of the anodic polarization and the corrosion potential of the inhibited electrode shifted toward the more noble potential by less than $45 \mathrm{mV}$ at the inhibitor concentration of $1 \times 10^{-3} \mathrm{M}$, as shown in Table 1. Thus, it is concluded that the inhibition of the anodic reaction is not attributed to the passivation of iron surface but to the adsorption of the inhibitor.

Some adsorption modes of the nitro compounds 


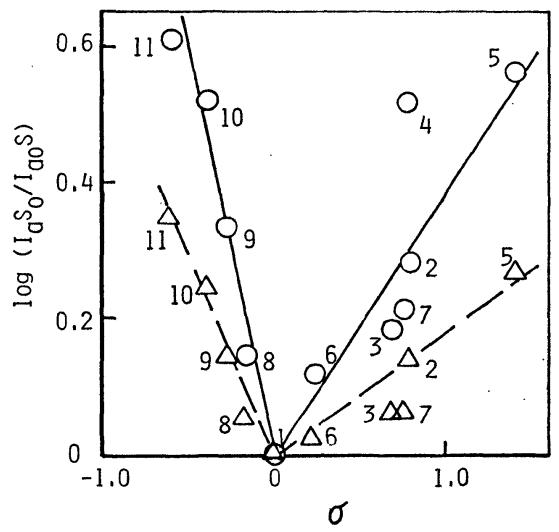

Fig. 5 Relationship between $\log \left(I_{a} S_{0} / I_{a 0} S\right)$ and Hammett constant, $\sigma$ at the inhibitor concentrations of $1 \times 10^{-3} \mathrm{M}(\mathrm{O})$ and $3 \times$ $10^{-3} \mathrm{M}(\triangle)$. The numbers refer to the inhibitors in Table 1.

on the iron surface were considered. Because the nitro group is electron-attracting, the compounds can adsorb on the surface by the electrostatic interaction between the metal and dipole. Fig. 4 shows the relationship between $I_{a} / S$ and dipole moment of the nitro compounds, $\mu$, exhibiting no evident correlation between them. From this result, it was concluded that the dipole-metal interaction is of a minor importance to the adsorption of nitro compounds.

Formation of a surface complex has been proposed by Riggs et al. for the adsorption of nitro compound on the titanium surface ${ }^{14)}$. The oxygen atoms of the nitro group attracting electrons from the phenyl group form the complex linkage with the titanium. If the nitro group plays an important role in the adsorption of the inhibitor, the inhibition efficiency should relate to a substituent constant.

According to the linear free energy relationship, if the activation energy of corrosion process is not changed by the adsorption of inhibitor and $I_{a}$ is low enough to be proportional to $S, I_{a}$ is expressed approximately by the equation

$$
\begin{aligned}
\log \left(I_{a} S_{0} / I_{a 0} S\right)= & \rho^{*} \Sigma^{*}+\delta E_{s} \\
& +\Psi_{r}+\alpha
\end{aligned}
$$

where zero subscripts refer to a reference inhibitor, $\Sigma \sigma^{*}$ and $E_{s}$ denote the polar and steric substituent constants, $\Psi_{r}$ is a parameter related to the electron-delocalized structures, and $\rho^{*}, \delta$, and $\alpha$ are constants, respectively ${ }^{2}$. Since all the inhibitors used in this study are benzene derivatives, the terms $\rho^{*} \Sigma \sigma^{*}$ and $\Psi_{r}$ are replaced by

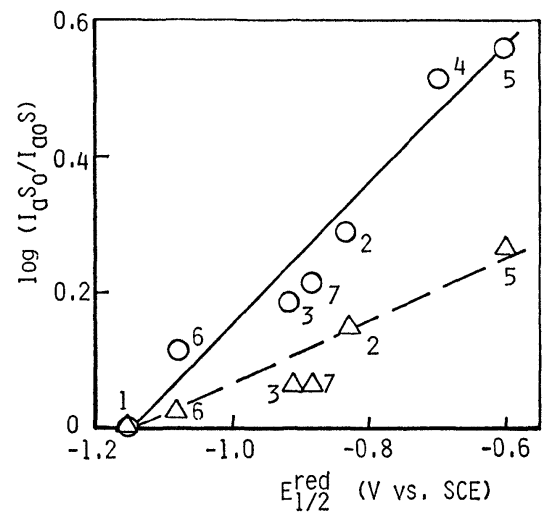

Fig. 6 Relationship between $\log \left(I_{a} S_{0} / I_{a 0} S\right)$ and half-wave potential of reduction, $E_{1 / 2}^{\text {red }}$ at the inhibitor concentrations of $1 \times 10^{-3} \mathrm{M}$ $(O)$ and $3 \times 10^{-3} \mathrm{M}(\triangle)$. The numbers refer to the inhibitors in Table 1.

$\rho \sigma$, the product of the reaction and Hammett constants. The term $\delta E_{s}$ which is related to the steric requirements for the adsorption of inhibitor was taken to be negligible. Therefore, Eq. (2) was written as

$$
\log \left(I_{a} S_{0} / I_{a 0} S\right)=\rho \sigma+\alpha
$$

The reference inhibitor was nitrobenzene.

The value of $\log \left(I_{a} S_{0} / I_{a 0} S\right)$ was plotted against $\sigma$ in Fig. 5, which showed two straight lines intersecting at the plot of nitrobenzene. For the nitro compounds in the range of $\sigma<0$, it is reasonable that the inhibitor chemisorbs on the metal by the electron donation of the nitro group to the metal orbital because an increase in the electron density of the nitro group enhances the inhibition efficiency. In the range of $\sigma>0$, however, the efficiency increases with a decrease in the density. This result cannot be explained by the chemical adsorption associated with the electron donation of the nitro group to the metal.

The inhibitors in the range of $\sigma>0$ are polynitro compounds except p-chloronitrobenzene. These inhibitor molecules can adsorb on the metal by the interaction between the plural functional groups and metal surface. The multi-functional adsorption results in the flat adsorption which is favorable for the $\pi$-electron bonding between the molecule and the metal.

The anodic inhibition efficiency of m-dinitrobenzene was lower than that of o- or p-dinitrobenzene, though the electron density in the nitro groups of the former molecule is higher by means of the resonance effects than the density in the groups of the latter two. This finding suggests 
the occurrence of adsorption by the different mode from the electron donation of inhibitor.

Since the electron-accepting inhibitor like the nitro compound can adsorb on the metal by the back-donation, the inhibition efficiency was confirmed to be correlated to the polarographic halfwave potential of reduction, $E_{1 / 2}^{\mathrm{red}}$ as a measure of the electron affinity. The value of $\log \left(I_{a} S_{0} / I_{a 0} S\right.$ was plotted in Fig. 6 against $E_{1 / 2}^{\mathrm{red}}$, which was determined in $0.1 \mathrm{M}$ tetraethyl ammonium perchlorate at $25^{\circ} \mathrm{C}$ by Peover ${ }^{15)}$. The figure exhibits a straight line at each of the concentrations and the increase of $I_{a}$ with the ability of electron acceptance. This result show that the chemisorption by the back-donation is of an importance to the adsorption of such the electron-accepting inhibitors as the nitro compounds.

The polar organic inhibitors like alkyl amines chemically adsorb by the donation of electrons in the polar group to the metal orbital, in general. Because of no electron-acceptable orbital in the molecule of the amines, the adsorption by the back-donation is impossible to occur. However, the inhibitors like anilines and olefinic amines, which can accept the electrons in the antibonding $\pi$-orbital, may readily adsorb by the back-coordination on the cathode. The possibility of the back-donation has also been shown in the previous studies on the cathoidc inhibition efficiency of these inhibitors ${ }^{2), 3)}$. The adsorption of these inhibitors by the donation of $\pi$-electrons to the metal orbital must be reinforced by the backdonation of metal electrons to the antibonding $\pi$-orbitals of the double bonds.

\section{Conclusion}

The polarization study was made on the iron electrode in the $\mathrm{HCl}$ solution inhibited with the nitrobenzene derivatives. These compounds accelerated the cathodic reaction because of their reduction, while they inhibited the anodic reaction. The inhibition efficiency of these inhibitors for the anodic reaction was correlated to the Hammett constant, $\sigma$ of substituent in the benzene ring. As for the compounds of which $\sigma$ is less than zero, the efficiency increased with the electron density of the nitro group. The efficiency of the compounds for $\sigma>0$ was enhanced with the decrease in the electron density. Because the efficiency of the latter compounds was correlated to the half-wave potential of reduction, the formation of inhibitor-metal bond by the back-donation was concluded to be a major factor for the adsorption of the nitro benzene derivatives.

(Received October 29, 1982)

\section{References}

1) O. L. Riggs, Jr.: "Corrosion Inhibitors", C. C. Nathan ed., p. 7, National Assoc. of Corr. Eng., Houston (1973).

2) K. Aramaki: Denki Kagaku, 46, 86 (1978).

3) K. Aramaki: Denki Kagaku, 43, 696 (1975).

4) K. Aramaki: "Proceedings 5th European Symposium on Corrosion Inhibitors", vol. 1, p. 267, Universita Degli Studi di Ferrara, Ferrara (1980); K. Aramaki, S. Iizumi \& F. Nakagawa: Boshoku Gijutsu, 29, 566 (1980).

5) K. Aramaki: Boshoku Gijutsu, 32, 144 (1983).

6) K. Aramaki: Denki Kagaku, 44, 771 (1976).

7) K. Aramaki \& M. Ichimura: Boshoku Gijutsu, 29, 437 (1980).

8) K. Aramaki \& N. Hackerman: J. Electrochem. Soc., 115, 1007 (1968).

9) K. Aramaki: Denki Kagaku, 42, 75 (1974).

10) A. P. Tomilov, S. G. Mairanovskii, M. Ya. Fioshin \& V. A. Smirnov: "Electrochemistry of Organic Compounds", p. 248, Halsted Press. New York (1972).

11) J. A. Petit, G. Chatainier \& F. Dabosi: "Proceedings 5th European Symposium on Corrosion Inhibitors", vol. 2, p. 413, Universita Degli Studi di Ferrara, Ferrara (1980); Corr. Sci., 21, 279 (1981).

12) F. Mansfeld \& J. V. Kenkel: Corr. Sci., 15, 767 (1975).

13) I. L. Rosenfeld: "Proceedings of the First Soviet-Japanese Seminar on Corrosion and Protection of Metals", Ya. M. Kolotyrkin ed. p. 86 Nauka, Moscow (1979).

14) O. L. Riggs, Jr., K. L. Morrison \& D. A. Brunsell: Corrosion, 35, 356 (1979).

15) M. E. Peover: Trans. Faraday Soc., 60, 479 (1962). 